\title{
Learning in Cooperating Agents Environment as a Method of Solving Transport Problems and Limiting the Effects of Crisis Situations
}

\author{
Jaroslaw Koźlak \\ Department of Computer Science, AGH-UST, Al. Mickiewicza 30, 30-059 Kraków, \\ Poland \\ kozlak@agh.edu.pl
}

\begin{abstract}
The realising of transport requests plays an important role for companies regarding cost. For this reason the construction of an optimal and effective transport planning and scheduling, offering the best use of transport means is hugely important. One of the researched transport problems at present is PDPTW. In this work, the PDPTW problem will be extended by adding changeable and uncertain travel times between given locations, which will then be examined and learnt by the dispatching company system. The changeable travel times are the results of traffic jams forming and propagating. The applied multi-agent approach allows us to consider the problem on the level of a whole company and on the local level analysed by a particular vehicle. The other aspects which are taken into consideration are traffic patterns, whose goal it is to facilitate the optimal routes for the given traffic patterns. These patterns contain the information about the dependencies between the traffic state on the particular routes.
\end{abstract}

Keywords: Multi-agent systems, transport problems, learning, traffic jams.

\section{Introduction}

Vast amounts of money from the budgets of many companies are spent on costs related to transport. In the case of some goods or services, these costs constitute the most dominant element. A lot of companies offer shipping services or services on demand, where it is necessary that the vehicle arrives at the destination in a given period of time. Additionally, and especially in the case of motor transport, it is difficult to predict the exact times of travel between locations. This could be a consequence of changing traffic volume, traffic jams or some other current unpredicted events. Therefore, the development of software tools which make route planning and predicting travel times possible, takes on a very high significance. This will also allow the limiting of the use of means of transport and total traversed distances, and will assure that the time and capacity constraints can be achieved. 
A lot of different transport problems like TSP, VRPTW or PDPTW in static and dynamic versions have been widely researched. The basis of the current work constitutes a dynamic version of the PDPTW (Pickup and Delivery Problem with Time Windows). The PDPTW consists of the realisation of a set of transport requests by a fleet of vehicles. In PDPTW, it is assumed that by using a fleet of vehicles it is necessary to perform a set of transport requests described by periods of time when operations of a pick up and a delivery may take place, locations where the goods are picked up and where they must be delivered as well as the quantity of commodities. The dynamic version of the problem concerns the requests arriving during the running of the system, a problem solving algorithm that must be able to be started repeatedly, the variable constraints related to time flow and performed operations of pickups and deliveries have to be taken into consideration as well as making it necessary for the movement of vehicles to be modeled.

In the version of the problem being analysed in this work, several extensions to dynamic PDPTW were introduced. They are based on assessing uncertain and changeable travel times between locations, which are additionally a result of the formation of traffic jams and them propagating onto neighbouring arcs. The problem, extended in such a way, better suits the situations we may encounter when planning the realisation of transport requests in practice. To solve the problem, the multi-agent approach has been chosen.

The layout of this paper is as follows. Section 2 contains an overview of the work being carried out within domains of transport planning and multi-agent systems. Section 3 concerns the presentation of a model of a multi-agent system for planning transport requests, while taking into consideration communication between agents, modelling and the spread of traffic jams and traffic patterns. Section 4 contains an overview of experiments carried out and the advantages offered by communication between agents as well as traffic patterns. Section 5 contains conclusions and plans for future research.

\section{State of the Art}

To solve transport planning and scheduling problems different approaches are applied. One can distinguish two major kinds of problems: static and dynamic. To solve the static problems nowadays, different heuristic approaches are usually used. They are based on algorithms such as evolutionary algorithms, simulated annealing, tabu search [5], squeaky wheel [6] or ant colony approach. To solve the dynamic problem, metaheuristics based on tabu search [4] or multiagent approaches are used. The multi-agent approach exploits methods such us Contract-Net, simulated trading [13] or DCSP [8].

The multi-agent approach facilitates an analysis of problems which consider additional elements that take part in the planning of transport requests in practice, but which are not present in the classical PDPTW. These aspects are for example modelling traffic jams [3], taking into consideration different kinds of 
freight and construction of the transport unit (composed of trucks and trailers adjusted to the given needs [2]) as well as a problem decentralisation [9].

The next aspect being analysed in this paper is a problem of uncertainty related to travel times through the arcs. This problem is examined in particular by the systems designed for city traffic modelling. A very popular approach which is the basis for different multi-agent realisations, is the Nagle-Schreckenberg model [7. Taking advantage of these systems and possessing information about city traffic, it is possible to estimate travel times in any given part of the route.

\section{Model}

The model presented in this work, besides solution optimisation methods focus on the learning of the travel times and recognition of traffic-jam patterns in the road network. The model of the system consists of a set of agents (a dispatcher and vehicles) and of the environment, where agents function (fig. 11).

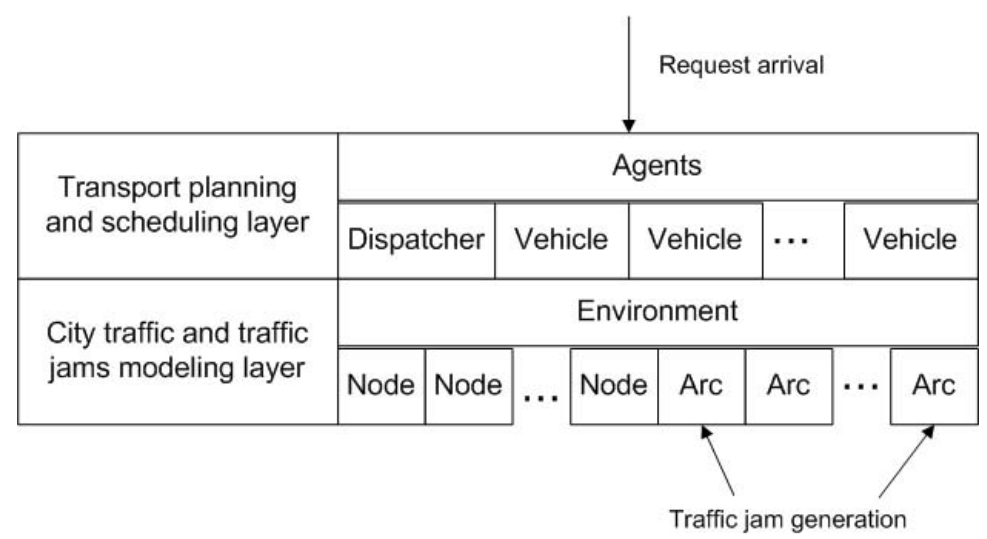

Fig. 1. System layers model

\subsection{Environment}

The environment is represented by the transport network, which is a graph $T N=(V, E)$, where $V$ is a set of nodes $\left(V_{i}\right)$ and $\mathrm{E}$ is a set of edges $\left(E_{i}\right)$. Each node is described by its coordinates $\left(x_{i}, y_{i}\right)$ and the probability $p g_{i}$ of a request generation taking this given node as a pickup or a delivery point. Each edge $E_{i}$ is described by nodes $V_{j}, V_{j^{\prime}}$ being the starting and ending nodes of the edge, the distance $d_{j j^{\prime}}$ between these nodes and a function of probability distribution $T_{i}$ which represents the travel times through the edge. The probability distribution function may vary depending on traffic volume on this edge $t v_{i}$. The traffic volume changes depending on the time of day or as a result of random events which cause the build-up of traffic (accidents, breakdowns, closing of roads). 


\section{$3.2 \quad$ Agent-Dispatcher}

The goal of the agent-dispatcher is to receive requests from customers and to assign these requests to the vehicles, according to the goal function. Additionally, agent-dispatcher manages a common knowledge of enterprise concerning any current appearances of traffic jams and patterns of traffic jam distribution.

The agent-dispatcher $A C$ is represented by the following parameters $\left(g_{A C}, R\right.$, $\left.R_{f}, R_{a}, R_{n}, R_{r}, K_{A V}, A_{r}, T J, T P\right)$ where: $g_{A C}$ - goal function of agent, $R$ set of arrived requests, $R_{f}, R_{a}, R_{n}, R_{r}$, - sets of requests realised, assigned to agent-vehicles to be realised, which have not yet been assigned for realisation to agent-vehicles and a set of rejected requests, $K_{A V}$ - set of information concerning the vehicles belonging to the company, $A_{r}$ - set of offers of request realisations, $T J, T P$ - knowledge about current traffic-jams and traffic patterns.

The knowledge bases $T J$ and $T P$ are made available as an external blackboard, where access is allowed for all agents - the agent-dispatcher as well as agents-vehicles. However, operations on them are performed by the dispatcher on the basis of data acquired from agents-vehicles.

The dispatcher may take info consideration different criteria of choice of vehicle, taking into consideration the preferences represented by goal function such as: as small as possible estimated costs related with the request realisation by the vehicle, or even distribution of requests among vehicles. The agent-dispatcher performs the following actions:

- actions related to an allocation of requests: receipt of request, bringing forward the requests to agents-vehicles, receipt of an offer from agent-vehicles, analysis of offers (the choice of the best offer of action realisation, clearing the set of offers of this request realisation and sending an answer of acceptance or rejection to agents which sent the propositions of request realisations), acceptance of a request to realisation or request rejection, receipt of information that a request has already been completed;

- actions related to management of knowledge base about the current trafficjams (receipt of information and table update);

- actions related to management of knowledge base about traffic patterns (receipt of information and table update).

\subsection{Agent-Vehicles}

The goal of agent-vehicle is to negotiate transport requests for realisation, determination using insertion algorithm to find the best positions in the travel route for adding new request points, request realisation and exploration of the road networks to improve the quality of information about travel times. Agent-vehicle $A V^{k}$ is represented by the following n-tuple $\left(V^{k}, R^{k}, R_{p}^{k}, R_{d}^{k}, P^{k}, P_{r}^{k}, \phi^{k}, p^{k}\right.$, $\left.g^{k}, c_{\text {max }}^{k}, c^{k}, s^{k}, A T, A T^{k}, r^{k}\right)$, where:

- $V^{k} \in \mathrm{V}$ - actual location (the node where the agent currently is at present or where it is currently aiming); 
$-R^{k}, R_{p}^{k}, R_{d}^{k}$ - set of requests assigned for realisation, picked up and have been realised (after delivery);

- $P^{k}$ - planned route, represented as a series $<p_{j}^{k}>$ where $p_{j}^{k}=V_{j}$;

- $P^{k}$ - planned travel route which consists only of pickup or delivery points;

$-\phi^{k}$ - function which assigns the request points to be performed at given locations to the points of the travel routes;

$-p^{k}-$ a special point in the travel route, describing the current position of the vehicle;

$-g^{k}-$ goal function describing the functioning of an agent;

$-c_{\max }^{k}-$ maximum capacity of vehicle;

$-c^{k}$ - current load, $c^{k}$ should be lower or equal to $c_{\max }^{k}$;

$-s^{k}$ - strategy which is used by the agent to estimate travel times between the nodes and to take into consideration the uncertainty of travel times (average value of estimations, given probability of successful plan realisation);

- AT - knowledge concerning the estimated travel times through the arcs;

- $A T^{k}$ - knowledge concerning travel times through the given arcs adjusted by the travel times coming from agent experiences (its times of travel through the arcs during its movements) and information obtained from other agents;

- $r^{k}$ - parameter describing an inclination to take a risk, for a small value of this parameter, the agent chooses the fastest known routes, for larger values, the agent tries to explore the road networks and to supplement the data about less explored arcs.

The quality of solution $g^{k}$ for the given vehicle $A V^{k}$ is described by eq. 1]

$$
g^{k}=\alpha \sum_{i=1}^{n} p_{i}-\beta c-\gamma \Sigma_{i=1}^{n}\left(f_{i}^{p}+f_{i}^{d}\right)
$$

where: $n$ - number of requests to be realised by a vehicle, $p_{i}$ - gain after realisation of request number, $c$ - costs caused by total distance travelled, $f_{i}^{p}, f_{i}^{d}-$ penalties caused by lateness at the points of pickup and delivery of request $i, \alpha$, $\beta, \gamma$ - weighting coefficients;

Agent-vehicle performs the following actions:

- actions related to request allocation: construction of route for a given set of requests taking into consideration information about travel times and a parameter describing the inclination to take a risk, route estimation, estimation of submitted proposition (it is described as a difference between goal function of agent after request acceptance and before request acceptance, the agent may also inform that it is not able to realise the request), submitting an offer of request realisation to agent-company or acceptance or rejection of request;

- actions related to vehicle movement: movement of vehicle, pickup realisation, delivery realisation;

- actions related to an updating of its own knowledge bases and those of other agents: modification of route because of traffic jam, modification of knowledge base about travel times through the $\operatorname{arcs} A T^{k}$, sending of message to agent-vehicles giving information on current travel times through a given arc, observation of existing traffic jams, acquiring information about traffic patterns. 


\subsection{Traffic Jams}

It is assumed that each arc has a maximum allowed capacity $c_{i j}^{\text {max }}$ and actual traffic $c_{i j}$. Additionally, arcs, coming out from a given node, are given weightings reflecting how the traffic coming into this node is divided among the arcs coming out from it. If the actual traffic reaches the values equal or greater than a maximum allowed capacity, a traffic jam will appear on these arcs, which will also slow down the movement of vehicles modelled on these arcs. A traffic jam may also be a result of a randomly generated crisis situation which increases the actual traffic volume on the arc. According to the rules of flow of traffic to neighbouring arcs, traffic jams may propagate.

If a vehicle reaches the start of an arc on which a traffic jam has appeared (it may be recognized by observing the relationship between the current traffic parameter and maximum allowed capacity on the arc), then the vehicle attempts to construct a diversion route. A route construction algorithm is initiated once more but in this case omitting the arc where the traffic jam has occurred. If this construction of a diversion is successful, the vehicle will then continue along this new modified route.

The distribution of travel times through the arcs are not known to the agents carrying out planning. The vehicles and dispatcher do not know the distribution on the given roads, they may only know some estimations and gain additional knowledge while obtaining their own experience or experience acquired by other vehicles. The agent may acquire information concerning travel times between the nodes using the following methods:

- as a result of traversing a given arc and making a measurement of the time required. In this case an estimated value of travel times between the nodes $i$ and $j$ is changed and takes a value equal to $t_{i j}^{(n+1)}=\alpha t_{i j}^{(n)}+\beta t_{i j}$, where $t_{i j}$ is the current travel time from $i$ to $j$, and $\alpha$ and $\beta$ are the weighting parameters, equal in its simple case to $n /(n+1)$ and $1 /(n+1)$, where $\mathrm{n}+1$ is a number of measurements taking the last one into consideration.

- as a result of obtaining information about travel times through the arc from the other cooperating agent. In this case, a new travel time estimation will be calculated in the similar manner as previously described and taken into consideration after during the planning process.

\subsection{Traffic Patterns}

The table concerning the traffic pattern is managed by an agent-dispatcher. Rows and columns are identified by the number of arcs $e_{j}$ and $e_{i}$. For each cell of this table, lists are stocked containing the gathered parameters describing results of observation of traffic states on these arcs measured within the tolerated time period: was a traffic jam observed on the arc $e_{i}$ (yes or no), was a traffic jam observed on the arc $e_{j}$ (yes or no), time of observation. This information is delivered to the agent-dispatcher which makes it accessible to particular agentvehicles afterwards. Agent-vehicle observing or obtaining information about a 
traffic-jam appearing on any given arc may verify whether a traffic-jam appearing on this arc was often associated with the appearances of traffic jams on other arcs contained in its planned route. If this is the case, the agent makes an attempt to construct a diversion route also omitting the arcs threatened by the appearance of traffic-jams.

\section{Experiments}

The experiments were conducted with the goal of estimating the quality of the system functionality depending on the selected behaviours of agents. In particular, the following aspects were considered:

- different frequencies of messages exchanged between agent-vehicles, these messages contained information about travel times through the arcs, the other option taken into consideration was the lack of exchange of this kind of information.

- application of traffic patterns which describe the dependencies between the state of traffic on the different pairs of arcs, the other option was to not take this element into consideration
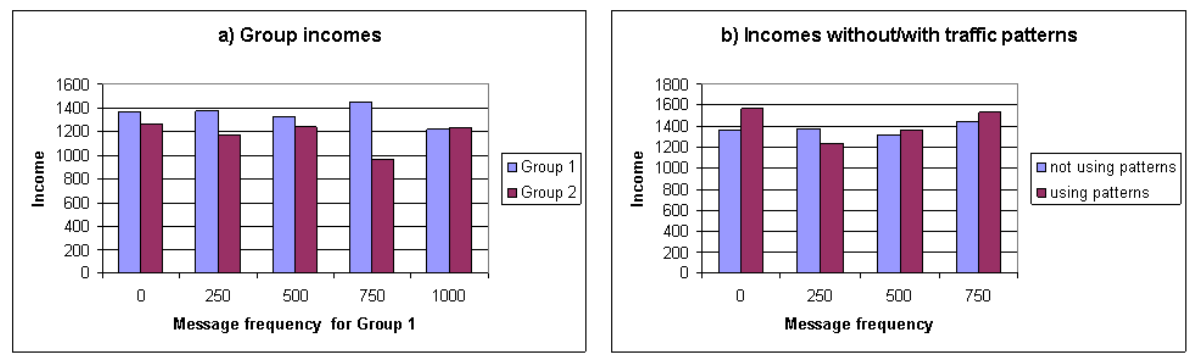

Fig. 2. Vehicle groups income a) different messages exchange frequencies - Group1 and no message exchange - Group2 b) with and without patterns

Vehicles in the system were divided into two groups. The vehicles in the first group exchange information about the travel times through the given arcs. The vehicles in the second group do not use this mechanism. As a method of estimating the quality of activities, the total income of vehicles in each group is considered. Experiments proved (fig. 4) that for almost each frequency of messages exchanged, the group which used this mechanism obtained better results. The only exception was a case when the communication had the lowest frequency. In this case the exchanged information might not be current and so has a limited influence on the improvement of the accuracy of traffic description on this arc. The results obtained for those vehicles using the patterns proved to give highest financial income than those that did not. Because the quantities of performed 
requests were similar in each case, the increase of income in the cases with communication and with the patterns is caused by a more accurate estimation of travel times which limits lateness and the consequent financial penalties.

\section{Conclusions}

The system for solving the transport problem as an extension to the dynamic PDPTW by uncertain travel times was realised. The experiments showed advantages resulting from the additional exchange of information among agents concerning travel times and the application of traffic patterns. Future work on the system will have as its goal the improvement of the quality of obtained solutions and to make verification better. In particular, an elaborate set of tests based on the benchmarks for static PDPTW is planned. The use of larger graphs representing the road networks, which is a real city (roads e.g. in Kraków.) is also planned.

Kind thanks to the Computer Science students at AGH-UST and the trainees who contributed to the work on the pilot version of the system and especially to M. Kwiecień, J. Rachwalik and Ch. Elmer.

\section{References}

1. Bachem, A., Hochstattler, W., Malich, M.: Simulated Trading A New Parallel Approach for Solving Vehicle Routing Problems. Proc. of Int. Conf. Parallel Computing: Trends and Applications, 1994.

2. Burckert, H.-J., Fischer, K., Vierke., G. : Transportation scheduling with holonic MAS - the TELETRUCK approach. Third International Conference on Practical Applications of Intelligent Agents and Multiagents (PAAM 98), 1998.

3. Fischer, K., Müller, J.P., Pischel, M.: Cooperative Transportation Scheduling: an Application Domain for DAI. Applied Artificial Intelligence, vol.10, 1996.

4. Gendreau, A., Guertin, F. , Potvin, J.Y., Séguin, R. :Neighborhood search heuristics for a dynamic vehicle dispatching problem with pick-ups and deliveries. Rapport technique CRT-98-10, Université de Montréal, 1998.

5. Li, H., Lim A.: A Metaheuristic for the Pickup and Delivery Problem with Time Windows. Proceedings of 13th IEEE International Conference on Tools with Artificial Intelligence (ICTAI'01), USA, 2001.

6. Lim, H. , Lim, A., Rodrigues, B.: Solving the Pick up and Delivery Problem using "Squeaky Wheel" Optimization with Local Search. Proceedings of American Conference on Information Systems, AMCIS 2002, USA.

7. Nagel, K., Schreckenberg, M.: A cellular automaton model for freeway traffic, J. Phisique I, 2(12), 1992.

8. Neagu, N., Dorer, K., Calisti, M.: Solving Distributed Delivery Problems with AgentBased Technologies and Constraint Satisfaction Techniques. Dist. Plan and Schedule Management, 2006 AAAI Spring Symp., The AAAI Press, USA.

9. Dorer, K., Calisti, C.: An Adaptive Solution to Dynamic Transport Optimization, Proceedings of the AAMAS05 industry track, Utrecht, The Netherlands, 2005. 